\title{
MAL DE POTT RÉVELÉ PAR UN ABCÈS LATÉROPHARYNGIEN CHEZ UN PILOTE
}

\author{
A. SETHOM, T. KHELIFI M. DARHMOUL, I. BEN DHIA, A. SOUISSI, I. DKHIL, S. BEN SALEM, R. \\ BOUATTOUR, C. BEN OTHMEN, C. JALEL *, N. GUERMAZI. \\ *SERVICE CHIRURGIE ORTHOPÉDIQUE HÔPITAL FSI LA MARSA \\ CENTRE DE MÉDECINE AÉRONAUTIQUE ET EXPERTISE DE TUNIS.
}

\begin{abstract}
RESUME
Introduction : La tuberculose rachidienne est la plus fréquente de la tuberculose ostéo-articulaire. Les auteurs rapportent un cas de mal de pott cervical avec abcès latéropharyngien.

Observation : IL s'agit d'un pilote âgé de 44 ans, présentant depuis 6 mois des névralgies cervicobrachiales droites et des dysphagies avec une perte de poids chiffrée à $5 \mathrm{~kg}$. L'examen ORL a révélé la présence d'un bombement modéré de la paroi postérolatérale droite de l'oropharynx s'étendant en bas jusqu'à l'hypopharynx. L'examen neurologique a retrouvé une limitation du mouvement d'abduction de l'épaule droite évoquant une parésie du nerf spinal et un réflexe bicipital droit aboli.

La TDM cervicale a révélé une collection latérocervicale droite étendue de C4 jusqu'à D1 et une lyse du corps de C6. L'IRM cervicale a conclue à l'absence d'anomalie du signal médullaire et disco-vertébral. La ponction biopsie du corps de C6 avait ramené du caséum dont l'analyse histologique a confirmé l'origine tuberculeuse. L'indication d'une exérèse chirurgicale du corps de C6 par abord antérieur a été posée devant la persistance de cervicalgies intenses. II a été ensuite mise en place une greffe iliaque de remplacement fixée par une plaque d'ostéosynthèse. Les suites opératoires étaient bonnes. Le traitement médical s'est basé sur les antituberculeux selon le protocole classique avec une durée totale de 12 mois. Après un recul moyen de trois ans, l'évolution a été jugée favorable avec une bonne consolidation du greffon et l'absence de récidive. Du point de vue aéronautique, le patient a été déclaré inapte définitif pour toutes les catégories du personnel navigant.

Conclusion : La tuberculose pose un problème de santé publique car elle sévit encore à l'état endémique. L'absence de spécificité clinique et radiologique nous amène à insister pour renforcer l'action de dépistage à tous les niveaux étant donné le nouveau profil de la maladie en Tunisie.
\end{abstract}

Mots clefs : Tuberculose, mal de Pott, abcès latéropharyngien.

\section{SUMMARY}

Introduction: Pott's disease is the most frequent localisation of osteoarticular tuberculosis. The authors report a case of cervical Pott's disease with lateropharyngeal abscess.

Observation: Our patient is a 44 years old pilot; he presented since 6 months cervicalgy, dysphagia and weighting loss. Clinical findings showed a swelling of posterior and lateral oropharyngeal wall extending to hypopharynx. A palsy of the $\mathrm{XI}$ th nerve and an abolition of the bicipital reflex were also noticed.

CT scan showed a lateropharyngeal collection extending from C4 to D1. It was also noticed a subtotal destruction of C6. However, there were no medullar lesions in the cervical spine MRI.

Tuberculosis was confirmed thanks to histological analysis of caseum taken during cervical biopsy.

The patient was operated using anterior approach. He had surgical removal of C6 which was replaced by iliac prothesis. Medical treatment was based on antituberculosis drugs used during 12 months. After three years of follow up, the out come was favourable with no recurrence and satisfying consolidation. For aeronautic considerations, the patient was declared unable for flying mission.

Conclusion: Tuberculosis constitutes a problematic infectious disease in Tunisia which may have serious consequences on publish health. We insist on preventing measurements because there are neither specific clinical findings nor radiological characterising features.

Keywords: Tuberculosis, Pott's disease, lateropharyngeal abscess

\section{INTRODUCTION}

La tuberculose est une maladie infectieuse spécifique causée par une mycobactérie : le bacille de Koch, elle est devenue plus rare en raison de la vaccination et de l'amélioration du cadre de la vie.

Les localisations extrapulmonnaires de la tuberculose sont peu fréquentes. Elles représentent 0,5 à $10 \%$ des manifestations cliniques de la maladie (1). Elles peuvent se présenter sous diverses formes cliniques. Nous rap- portons un cas de spondylodiscite tuberculeuse C5-C6 associée à un abcès latéropharyngien survenant chez un pilote professionnel suivi au centre de médecine aéronautique et d'expertise de Tunis.

\section{OBSERVATION :}

II s'agit d'un patient âgé de 44 ans, pilote de transport (avion à hélice) depuis vingt ans, régulièrement suivi en visite révisionnelle au centre d'expertise de médecine 
aéronautique de Tunis. Le patient a été correctement vacciné par le BCG et il est HIV négatif. II a présenté depuis 6 mois des cervicalgies récidivantes associées à des douleurs pharyngées avec une odynophagie et une perte de poids chiffrée à $5 \mathrm{~kg}$. L'interrogatoire révèle la présence de névralgies cervicobrachiales droites de topographie 66 et l'installation d'un torticolis récent.

L'examen ORL a révélé la présence d'un bombement régulier sous muqueux modéré de la paroi postérolatérale droite de l'oropharynx s'étendant en bas jusqu'à l'hypopharynx et une adénopathie spinale droite de $2,5 \mathrm{~cm}$. L'examen neurologique a retrouvé une limitation du mouvement d'abduction de l'épaule droite et une abolition du réflexe bicipital à droite témoignant d'une atteinte C5-C6. Le bilan biologique a révélé une accélération de la vitesse de sédimentation à 102 à la première heure. L'intradermoréaction (IDR) à la tuberculine était positive. Mais la bacilloscopie était négative, infirmant l'atteinte pulmonaire primitive.

L'indication d'une ponction transorale de la tuméfaction oropharyngée fût posée mais le patient a refusé.

La TDM cervicale a permis de révéler une collection latérocervicale droite étendue de C3 à D1, nécrosée en son centre évoquant un abcès latéropharyngien (figures 1, 2).

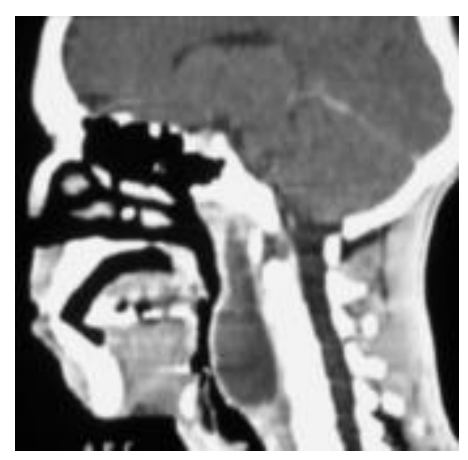

Figure 1 : TDM cervicale en reconstruction coronale montrant l'abcès rétro latéropharyngien droit.

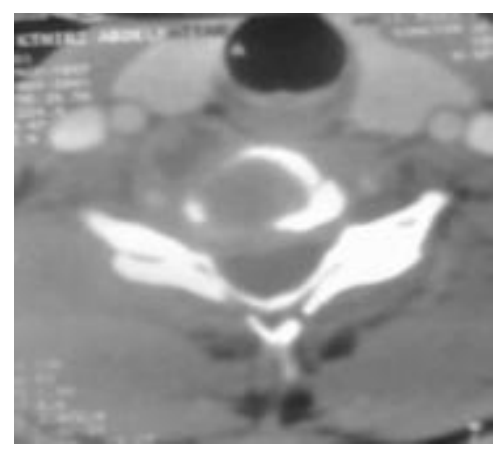

Figure 2 : TDM cervicale en coupe axiale montrant l'abcès latéropharyngien droit et la lyse de $\mathbf{C 6}$.

II s'y associait une spondylodiscite C5-C6 et une lyse de la partie droite du corps de C6 (figures 3, 4, 5, 6). L'IRM cervicale a conclue à l'absence d'anomalie de signal disco-vertébral ainsi qu'à l'absence d'anomalie de signal de la moelle et des espaces périmédullaires.

Le patient a été hospitalisé en neurochirurgie où une ponction biopsie du corps de C6 a été réalisée, par un abord antérieur du rachis cervical ramenant du caséum. L'étude anatomopathologique et bactériologique de la nécrose caséeuse prélevée a confirmé l'atteinte tuberculeuse. (fig.3-4-5-6)
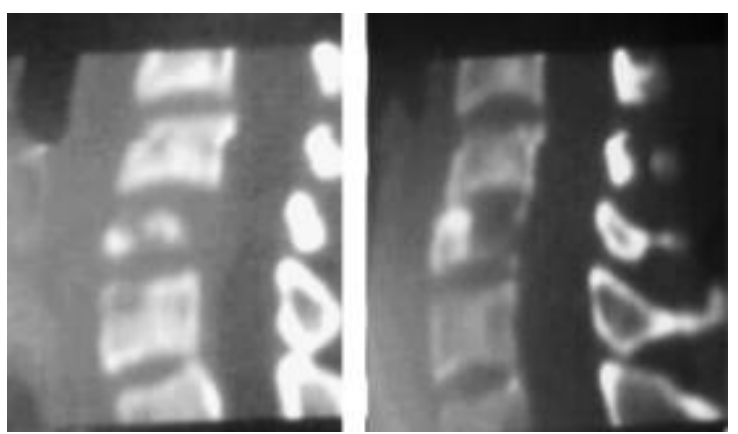

Figures 3 et 4 : TDM Cervicale rachidienne en reconstruction sagittale : la lyse de $\mathrm{C} 6$
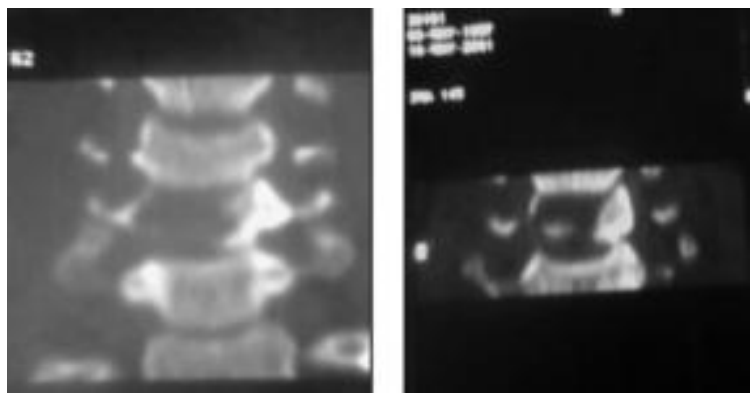

Figures 5 et 6 : TDM cervicale rachidienne (reconstruction): lyse subtotale du corps de C6.

Après un bilan préthérapeutique, le malade a reçu un traitement médical à base d'antituberculeux majeurs associant deux mois de traitement quadruple à base d'INH à la dose de $3 \mathrm{mg} / \mathrm{kg} / \mathrm{j}$, de rifampicine à la dose de $10 \mathrm{mg} / \mathrm{kg} / \mathrm{j}$, d'ethambutol à la dose de $20 \mathrm{mg} / \mathrm{kg} / \mathrm{j}$ et de la streptomycine ( un gramme par jour ) avec un contrôle mensuel de la fonction hépatique. Cependant la persistance de douleurs cervicales intenses devenant insomniantes, expliquées par la lyse importante de C6 et par le tassement discovertebral, a fait poser l'indication d'une exérèse chirurgicale du corps de C6. Le patient a donc été opéré par abord antérieur après un délai d'évolution de six mois .IL a bénéficié d'une mise en place d'une greffe iliaque de remplacement fixée par une plaque d'ostéosynthèse. Les suites opératoires étaient bonnes. Une immobilisation par une minerve cervicale a été préconisée en vue d'une contention post opératoire pendant une 
durée de deux mois. Le relais du traitement quadruple s'est fait ensuite par l'association INH et rifampicine en prise quotidienne. La durée totale du traitement médical était de 12 mois.

En post opératoire, l'évolution locale a été jugée favorable avec une bonne consolidation du greffon et l'absence de récidive. Au niveau de la sphère ORL, l'évolution s'est faite vers la disparition du bombement pharyngien juste après la chirurgie. Le ganglion cervical a disparu après quatre mois du début du traitement antituberculeux. Sur le plan neurologique, le patient a récupéré une mobilité normale dans le territoire $\mathrm{C} 5$ et $\mathrm{C} 6$ après un délai post-opératoire de cinq mois.

Du point de vue aptitude aéronautique, le patient a été déclaré inapte définitif pour toutes les catégories du personnel navigant étant donné que les lésions séquellaires du bloc vertébral cervical ne lui permette plus d'assumer en toutes sécurité ses missions de pilote.

\section{DISCUSSION :}

La tuberculose pose un problème de santé publique en Tunisie car elle sévit encore à l'état endémique (1). Actuellement, elle connaît une recrudescence nette de part le monde, vu l'apparition de l'infection par le HIV qui était responsable de cette flambée. Ce phénomène ne semble pas retentir sur l'incidence de la maladie dans notre pays (1).

La tuberculose extrapulmonnaire est dominée par l'atteinte ganglionnaire ; les localisations primitives extraganglionnaires sont les plus rares $(1,2,3)$. L'un des aspects originaux de notre observation est qu'elle décrit un nouveau cas de spondylodiscite tuberculeuse révélée par un abcès latéropharungien.

Du point de vu physiopathogénique, l'inoculation par le BK peut se faire de différentes façons $(1,2)$. La dissémination par voie hématogène se fait à partir d'un site contaminé surtout pulmonaire. La contamination par les expectorations bronchiques définie la voie canalaire aérique. Enfin l'infection peut se propager par voie lymphatique à partir d'un foyer cutané ou muqueux.

Cette diversité des voies de contamination explique la multiplicité de la topographie lésionnelle.

Parmi les localisations ORL, la tuberculose rhinopharyngée est la plus fréquente et c'est celle qui a fait l'objet de plus de publications dans la littérature (2). L'atteinte oropharyngée est souvent amygdalienne $(3,4)$.Les adénopathies cervicales sont quasi constantes $(1,2,3)$.

L'abcès latéro ou rétropharyngé constitue un mode classique de révélation d'un mal de pott cervical, son incidence varie de 57 à $70 \%$ (5). Selon KOOLI (5), la tuberculose vient à la tête des étiologies des abcès froids parapharyngiens. Le tableau clinique est alors souvent fait de cervicalgies, de dysphagies voir même de névralgies cervicobrachiales $(5,6)$. Moalla $\mathrm{J}$ rapporte un cas tunisien de mal de Pott sous occipital révélé par un abcés rétropha- ryngien (6).

$\mathrm{Au}$ cours de la tuberculose ostéo-articulaire, l'atteinte rachidienne représente la localisation dominante (7). Elle réalise plusieurs tableaux dont le plus habituel est l'atteinte discovertébrale ou mal de Pott. L'atteinte centrosomatique et vertébrale postérieure sont rares et sources de difficultés diagnostiques $(7,8)$. La traduction clinique est souvent insidieuse expliquant le retard diagnostique, pouvant être source de complications neurologiques. En effet, La gravité de cette localisation ostéovertébrale réside dans le risque de survenue de ces complications dans 10 à $47 \%$ des cas, pouvant aller de la simple

modification des réflexes ostéotendineux à la paraplégie $(5,7)$. L'imagerie par résonnance magnétique et la Tomodensitométrie constituent des examens de choix pour le diagnostic de la spondylodiscite tuberculeuse $(1,8,9)$. Selon Le Page (9), l'association de lésions vertébroépidurales postérieures à des lésions nécrosées sous formes d'abcès constituent le meilleur argument radiologique en faveur de la tuberculose.

L'IDR à la tuberculine reste un bon moyen de présomption mais son résultat n'est significatif que si le sujet est jeune et non vacciné $(1,2,6)$.

Cependant, la certitude diagnostique de la tuberculose tout siège confondu n'est apportée que par la confirmation anatomopathologique. L'aspect histologique est pathognomonique devant la constatation d'une nécrose caséeuse associée à un granulome épithéloïde gigantocellulaire.

Comme toute localisation extrapulmonnaire, le traitement est médical faisant appel aux antituberculeux usuels selon le protocole classique. La durée totale du traitement est variable de dix à dix huit mois selon les auteurs $(8,10,11)$. Cependant elle est moyenne de douze mois. La topographie lésionnelle ostéovertébrale fait prolonger le traitement selon certains jusqu'à dix huit mois $(8,11)$. L'absence d'amélioration clinique doit faire évoquer une erreur diagnostique ou un problème de résistance du BK aux antibacillaires $(1,2,11)$. En effet, on assiste actuellement à une émergence de souches mutantes qui sont à l'origine de phénomènes de résistance de plus en plus fréquents d'où l'importance d'une stratégie thérapeutique adaptée et généralisée pour toutes les formes primitives extrapulmonnaire quelque soit leur localisation.

Les indications de chirurgie dans le traitement de la tuberculose ostéoarticulaire ont considérablement diminué en raison de l'efficacité de la chimiothérapie antibacillaire. Le traitement médical peut alors être commencé trois semaines avant l'intervention et être prolongé 6 à 8 mois après (11). La chirurgie est indiquée s'il ya une compression médullaire ou à cause de lésions vertébrales destructrices sévères entraînant une déformation et / ou une importante instabilité. En cas d'abcès paravertébrale qui ne régresse pas en dépit du traitement médical, la chirurgie est pratiquée afin de permettre un drainage et une 
excision-parage des foyers nécrotiques tissulaires et des séquestres osseux en vers lesquels la diffusion des antituberculeux est insuffisante pour assurer la guérison totale $(5,6,11)$.

Par ailleurs notre observation présente des particularités aéronautiques vu le contexte professionnel de notre patient. En effet, il s'agit d'un pilote d'avion à hélice régulièrement exposé aux vibrations et constamment exposé aux facteurs de charges aéronautiques. Cela risque d'aggraver les lésions séquellaires du bloc vertébral cervical opéré et de provoquer des altérations discovertébrales surajoutées.

L'état de santé de notre patient certes atteint d'une maladie infectieuse bénigne, la tuberculose, lui a tout de même value une inaptitude définitive à l'exercice de ses fonctions de navigant après une vingtaine d'années de service. C'est dire l'importance de confirmer le diagnostic le plus rapidement possible et de traiter efficacement cette pathologie qui sévit toujours sous nos climats.

\section{CONCLUSION}

L'originalité de notre observation est qu'elle rapporte une forme de tuberculose à topographie lésionnelle peu commune chez un pilote professionnel. En effet, les atteintes latéropharyngées et cervicales rachidiennes constituent l'une des manifestations cliniques extrapulmonnaires les plus rares de la maladie. L'absence de spécificité clinique et radiologique nous amène à insister sur le diagnostic positif précoce étant donné le nouveau profil épidémiologique et bactériologique de la tuberculose en Tunisie.

\section{REFERENCES}

1. Ouni $\mathrm{H}$, Akkari $\mathrm{K}$, Sethom A et al. Tuberculose ORL extraganglionnaire ; à propos de 11 cas. J Tun ORL $2005 ; 23-5$.

2. Karsten M. Mycobacterial infections the head and neck. Otolaryngol clin N Am 2003; 36: 569-76.

3. Kharoubi S. La tuberculose pharyngée : étude analytique, à propos de 10 cas. Rev Laryngo Otol Rhino $1999 ; 119: 203-7$.

4. Moulonguet $L$, Delguidice $P$, Chauvin JL. L'Angine tuberculeuse : à propos d'un cas au Senégal. Ann Oto-Laryng $1995 ;$ 112:78-9

5. Kooli H, Marrakchi M, Tiss M et al. Cold Parapharyngeal abcess in spondilodiscits. Presse med 2001,30:19-21.

6. Moalla J, Mnejja S, Kallel S et al. Mal de Pott sous occipital révélé par un abcès rétropharyngien. $\mathrm{J}$ tun $\mathrm{ORL} 2008 ; 41-4$

7. Bernard L, Perrone C. La tuberculose ostéoarticulaire aujourd'hui. Presse Med 1997, $26: 308-10$.

8. Sahli $\mathrm{H}$, Ajlani $\mathrm{H}$, Landolsi $\mathrm{H}$ et all. La tuberculose de l'arc postérieur vértébral. La Tunisie Médicale 2008 ; 86 :939-40.

9. Le Page, Feydy A, Lefort A et al . Mal de pott cervical valeur de l'IRM et du scanner dans le bilan lesionnel. Rev Med int 2003, $24: 4-12$.

10. Achouri M, Hilmani $\mathrm{S}$, Lakhdar $\mathrm{H}$ et al. abord antérieur du rachis cervical dans le mal de pott. Rev chir orthop 1997,84:447-53.

11. Annabi H., Abdelkafi M., Trabelsi M. Tuberculose ostéoarticulaire mise au point. Tun Orthop $2008 ; 1(1): 1-8$ 